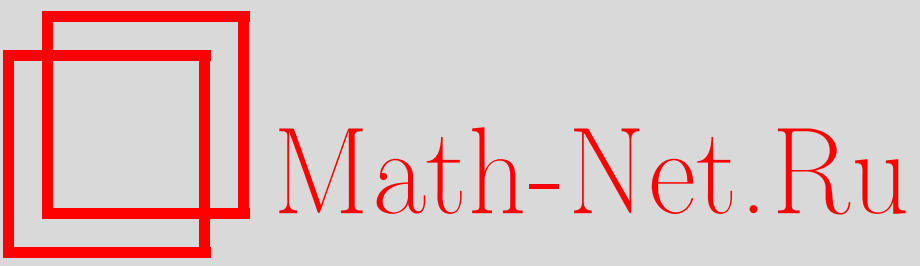

В. П. Маслов, О. Ю. Шведов, О начальных условиях в квазиклассической теории поля, ТМФ, 1998, том 114, номер 2, 233-249

DOI: https://doi.org/10.4213/tmf837

Использование Общероссийского математического портала Math-Net.Ru подразумевает, что вы прочитали и согласны с пользовательским соглашением

http://www . mathnet.ru/rus/agreement

Параметры загрузки:

IP : 54.172 .240 .79

26 апреля 2023 г., 13:24:28 
ТЕОРЕТИЧЕСКАЯ

И МАТЕМАТИЧЕСКАЯ

ФИЗИКА

Том 114, № 2

февраль, 1998

(C) $\mathbf{1 9 9 8}$ г.

В.П. Маслов*, О. Ю. Шведов*

\section{О НАЧАЛЬНЫХ УСЛОВИЯХ \\ В КВАЗИКЛАССИЧЕСКОЙ ТЕОРИИ ПОЛЯ}

Изучается проблема расходимостей и перенормировок в гамильтоновом формализме квазиклассической теории поля. Известно, что при таком подходе в главном порядке разложения могут возникать расходимости, для устранения которых предлагалось использовать неэквивалентные представления канонических коммутационных соотношений в различные моменты времени. В работе рассматривается уравнение Шредингера с ультрафиолетовым и инфракрасным обрезаниями. Для перехода к снятию обрезаний накладываются, помимо условий на контрчлены в гамильтониане, условия на начальное состояние регуляризованной теории. В главном порядке квазиклассического разложения приводится явный вид этих условий, инвариантный относительно эволюции. Тем самым показывается, что в этом приближении нет необходимости рассматривать неунитарные преобразования эволюции.

\section{1. ВВЕ ДЕНИЕ}

В квантовой теории поля часто используется квазиклассическое приближение. Примерами, в частности, являются:

1) квантование в окрестности солитонов - решений классических уравнений поля $[1-3]$;

2) исследование процессов в сильных электромагнитных и гравитационных полях [4-7];

3) гауссовское приближение, рассмотренное в работах [8-10].

Под квазиклассической теорией поля понимается обычно следующее. Поле $\varphi$, входящее в лагранжеву теорию поля с функционалом действия вида

$$
\frac{1}{g} S[\varphi \sqrt{g}]
$$

( $g$ - "малый параметр"), раскладывается на две части - "классическую", которая имеет порядок $1 / \sqrt{g}$, и “квантовую". Таким образом,

$$
\varphi=\Phi_{c} / \sqrt{g}+\phi .
$$

\footnotetext{
* Московский государственный университет, Москва, Россия
} 
Подстановка этого разложения в действие (1) приводит в главном порядке по $g$ к квадратичному действию

$$
\frac{1}{2} \phi \frac{\delta^{2} S}{\delta \Phi_{c} \delta \Phi_{c}} \phi+\text { const }
$$

т.к. линейное по $\phi$ слагаемое обрашается в нуль в силу классических уравнений движения.

Поскольку теория поля (3) является теорией с квадратичным действием, соответствуюшее квантовое уравнение Шредингера содержит гамильтониан, квадратичный по операторам рождения и уничтожения $a^{ \pm}(\mathbf{k})$ частиц с импульсом $\mathbf{k}$ :

$$
\widehat{H}_{2}=\int d \mathbf{k}\left[\frac{1}{2} a^{+}(\mathbf{k})\left(\widehat{A}^{t} a^{+}\right)(\mathbf{k})+a^{+}(\mathbf{k})\left(\widehat{B}^{t} a^{-}\right)(\mathbf{k})+\frac{1}{2} a^{-}(\mathbf{k})\left(\widehat{A}^{t *} a^{-}\right)(\mathbf{k})\right]+\gamma^{t} .
$$

Здесь $\widehat{A}^{t}$ и $\widehat{B}^{t}$ - операторы в пространстве функций переменной $\mathbf{k}$, зависяшие от времени в случае, если классическое решение $\Phi_{c}$ несташионарно; $\gamma^{t}$ - числовая функция. Уравнение Шредингера с квадратичным гамильтонианом решается точно [11].

При таком рассмотрении не учитывается проблема расходимостей в квантовой теории поля. Это приводит к тому, что в рассматриваемой квадратичной теории каноническое преобразование, связываюшее операторы рождения и уничтожения в разные моменты времени, в ряде случаев не является унитарным $[4,12]$.

Действительно, рассмотрим выражение

$$
\text { const } \exp \left(\frac{1}{2} \int d \mathbf{k} a^{+}(\mathbf{k})\left(\widehat{M}^{0} a^{+}\right)(\mathbf{k})\right) \Phi^{(0)},
$$

где $\widehat{M}^{0}$ - оператор с симметричным ядром, действующий в пространстве функций векторной переменной $\mathbf{k}$. Норма $\widehat{M}^{0}$ меньше единицы, $\Phi^{(0)}$ - вакуумный вектор пространства Фока. Известно [11], что данное выражение определяет элемент фоковского пространства тогда и только тогда, когда оператор $\widehat{M}^{0}$ является оператором ГильбертаШмидта. Решение уравнения Шредингера для гамильтониана (4), удовлетворяюшее начальному условию (5), имеет в момент времени $t$ вид

$$
c^{t} \exp \left(\frac{1}{2} \int d \mathbf{k} a^{+}(\mathbf{k})\left(\widehat{M}^{t} a^{+}\right)(\mathbf{k})\right) \Phi^{(0)}
$$

с зависящей от времени константой $c^{t}$ и оператором $\widehat{M}^{t}$, удовлетворяющим уравнению

$$
i \dot{\widehat{M}}^{t}=\widehat{A}^{t}+\widehat{B}^{t} \widehat{M}^{t}+\widehat{M}^{t}\left(\widehat{B}^{t}\right)^{T}+\widehat{M}^{t} \widehat{A}^{t *} \widehat{M}^{t}
$$

Оказывается, что $\widehat{M}^{t}$ необязательно является оператором Гильберта-Шмидта, даже если $\widehat{M}^{0}$ обладает этим свойством. Таким образом, уравнение Шредингера не имеет в этом случае решений, принадлежаших пространству Фока. 
Данный пример послужил одним из аргументов в пользу того, что преобразование эволюции в квантовой теории поля неунитарно и в разные моменты времени следует использовать разные представления канонических коммутационных соотношений $[12,13]$.

Мы покажем, что, по крайней мере в главном порядке квазиклассического разложения, нет необходимости рассматривать неунитарные преобразования эволюции. Оказывается, что элементы пространства Фока квадратичной теории, вообше говоря, не отвечают однозначно элементам пространства $\mathcal{H}_{\text {ren }}$ перенормированной теории. Мы покажем также, что обобщенный вектор (5) отвечает элементу пространства $\mathcal{H}_{\text {ren }}$ не тогда, когда оператор $\widehat{M}^{0}$ является оператором Гильберта-Шмидта, а при выполнении более сложного условия, которое и будет получено в данной работе.

Рассмотрим уравнение Шредингера, зависяшее от параметров ультрафиолетового и инфракрасного обрезаний $\Lambda$ и $L$,

$$
i \dot{\Psi}_{\Lambda, L}^{t}=H_{\Lambda, L} \Psi_{\Lambda, L}^{t}
$$

$\Psi_{\Lambda, L}^{t}-$ вектор гильбертова пространства $\mathcal{H}$. При определенных условиях на "контрчлены", входящие в гамильтониан $H_{\Lambda, L}$, величины типа функций Грина или $S$-матрищы не будут содержать в каждом порядке теории возмушений расходимостей при $\Lambda, L \rightarrow \infty$. Однако для исследования эволюции системы на конечных временах необходимо, помимо условий на гамильтониан, накладывать также условия и на начальные данные для уравнения (8).

В конструктивной теории поля [14-17] переход от регуляризованной теории к локальной осушествляется обычно следуюшим образом. Рассматриваются "перенормирован-

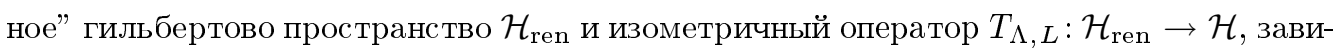
сяший от параметров ультрафиолетового и инфракрасного обрезаний. Обозначим через $U_{\Lambda, L}^{t}$ оператор эволюции в регуляризованной теории (8). Оператор $T_{\Lambda, L}$, называемый обычно “одевающим преобразованием", выбирается таким образом, чтобы операторы

$$
W_{\Lambda, L}^{t}=\left(T_{\Lambda, L}\right)^{-1} U_{\Lambda, L}^{t} T_{\Lambda, L}
$$

сильно сходились при $\Lambda, L \rightarrow \infty$ на некоторой плотной области определения. Предельный оператор $\lim _{\Lambda, L \rightarrow \infty} W_{\Lambda, L}^{t}$ называется оператором эволюции в перенормированной теории.

Данное определение означает, что если начальное условие для уравнения (8) выбрано в виде

$$
T_{\Lambda, L} X
$$

где $X$ - не зависящий от $\Lambda, L$ элемент пространства $\mathcal{H}_{\mathrm{ren}}$, то и решение этого уравнения записывается в аналогичном виде: $T_{\Lambda, L} X^{t}+Z_{\Lambda, L}^{t}$, где "невязка" $Z_{\Lambda, L}^{t}$ стремится по норме пространства $\mathcal{H}$ к нулю при $\Lambda, L \rightarrow \infty$.

Отметим, что если выражения $\left(T_{\Lambda, L}\right)^{-1} a^{ \pm}(\mathbf{k}) T_{\Lambda, L}$ задают операторнозначные обобщенные функции, имеюшие пределы при снятии обрезаний, то преобразование $T_{\Lambda, L}$ позволяет построить, вообще говоря, нефоковское представление канонических коммутационных соотношений. 
Однако существование преобразований $T_{\Lambda, L}$ доказано только для ограниченного класса моделей в двумерном и трехмерном пространстве-времени [14-17]. Для моделей обшего вида преобразование $T_{\Lambda, L}$ строится в виде формального степенного ряда теории возмушений по $g^{1 / 2}[15,18,19]$. При этом доказывается, что оператор (9) регулярен в рамках теории возмушений. Это означает, что коэффициентные функции разложения этого оператора в ряд теории возмушений имеют сильный предел.

Ниже будет построено преобразование $T_{\Lambda, L}$ в главном порядке квазиклассического разложения, в этом приближении найден вид вектора (10) регуляризованной теории и рассмотрен переход к снятию регуляризации.

\section{2. КВАЗИКЛАССИКА В РЕГУЛЯРИЗОВАННОЙ ТЕОРИИ ПОЛЯ}

В этом разделе мы приведем явный вид квазиклассического асимптотического разложения в теории поля, ограничиваясь для простоты рассмотрением одного скалярного поля с действием

$$
S[\varphi, g]=\int d x\left[\frac{1}{2} \partial_{\mu} \varphi \partial^{\mu} \varphi-\frac{m^{2}}{2} \varphi^{2}-\frac{1}{g} V_{\mathrm{int}}(\sqrt{g} \varphi)\right],
$$

где $x=\left(x^{0}, \ldots, x^{d}\right), \quad V_{\mathrm{int}}(\Phi)=O\left(\Phi^{3}\right), \partial_{\mu}=\partial / \partial x^{\mu}$. Другие модели теории поля рассматриваются аналогично. Для исследования расходимостей будем рассматривать вместо действия (11) действие, представленное в виде суммы

$$
S_{\Lambda, L}^{0}(\varphi, g)+S_{\Lambda, L}^{\mathrm{ct}}(\varphi, g)=S_{\Lambda, L}^{0}(\varphi, g)+\sum_{n \geq 1} g^{n-1} S_{\Lambda, L}^{n}(\varphi \sqrt{g})
$$

классического действия $S_{\Lambda, L}^{0}$ с ультрафиолетовым и инфракрасным обрезаниями

$$
S_{\Lambda, L}^{0}[\varphi, g]=\int d x\left[\frac{1}{2} \partial_{\mu} \varphi \partial^{\mu} \varphi-\frac{m^{2}}{2} \varphi^{2}\right]-\int d x \chi(\mathbf{x} / L) \frac{1}{g} V_{\mathrm{int}}\left(\sqrt{g} \varphi^{\Lambda}\left(x^{0}, \mathbf{x}\right)\right)
$$

и контрчленного действия $S_{\Lambda, L}^{\mathrm{ct}}, n$-е слагаемое которого $S_{\Lambda, L}^{n}$ отвечает расходимостям в $n$-петлевых диаграммах Фейнмана. Функции

$$
\varphi^{\Lambda}(t, \mathbf{x})=\int d \mathbf{y} \rho(\Lambda(\mathbf{x}-\mathbf{y})) \varphi^{\Lambda}(t, \mathbf{y})
$$

$\chi$ и $\rho$ пренадлежат пространству Шварца $\left(\chi(0)=1, \int d \mathbf{z} \rho(\mathbf{z})=1\right)$. При $\Lambda, L \rightarrow \infty$ действие $S_{\Lambda, L}^{0}$ переходит в (11).

Классический гамильтониан, отвечающий действию (12), представляется в виде $H_{\Lambda, L}=H_{\Lambda, L}^{0}+H_{\Lambda, L}^{\mathrm{ct}}$ функционала полей $\varphi(\mathbf{x})$ и импульсов $\pi(\mathbf{x})$, где

$$
\begin{aligned}
& H_{\Lambda, L}^{0}=\frac{1}{g} H_{\Lambda, L}^{(0)}[\pi \sqrt{g}, \varphi \sqrt{g}]=\frac{1}{g} H_{0}[\pi \sqrt{g}, \varphi \sqrt{g}]+\frac{1}{g} H_{\Lambda, L}^{\mathrm{int}}[\pi \sqrt{g}, \varphi \sqrt{g}], \\
& H_{0}[\Pi(\cdot), \Phi(\cdot)]=\int d \mathbf{x}\left[\frac{1}{2} \Pi^{2}(\mathbf{x})+\frac{1}{2}(\nabla \Phi)^{2}(\mathbf{x})+\frac{m^{2}}{2} \Phi^{2}(\mathbf{x})\right], \\
& H_{\Lambda, L}^{\mathrm{int}}[\Pi(\cdot), \Phi(\cdot)]=\int d \mathbf{x} \chi(\mathbf{x} / L) V_{\mathrm{int}}\left(\Phi^{\Lambda}(\mathbf{x})\right) .
\end{aligned}
$$


Контрчленный гамильтониан зависит от малого параметра:

$$
H_{\Lambda, L}^{\mathrm{ct}}=\sum_{n \geq 0} g^{n} H_{\Lambda, L}^{(n+1)}[\pi \sqrt{g}, \varphi \sqrt{g}]
$$

Конкретный вид контрчленов будет дан ниже.

Квантовое уравнение Шредингера для вектора $\Psi_{\Lambda, L, g}^{t}$ пространства Фока $\mathcal{H}$ имеет вид

$$
i \frac{d}{d t} \Psi_{\Lambda, L, g}^{t}=\sum_{n \geq 0} g^{n-1} H_{\Lambda, L}^{(n)}[\sqrt{g} \widehat{\pi}(\cdot), \sqrt{g} \widehat{\varphi}(\cdot)] \Psi_{\Lambda, L, g}^{t},
$$

где

$$
\begin{aligned}
& \widehat{\pi}(\mathbf{x})=\frac{i}{(2 \pi)^{d / 2}} \int d \mathbf{k} \sqrt{\frac{\omega_{\mathbf{k}}}{2}}\left[a^{+}(\mathbf{k}) e^{-i \mathbf{k} \mathbf{x}}-a^{-}(\mathbf{k}) e^{i \mathbf{k} \mathbf{x}}\right] \\
& \widehat{\varphi}(\mathbf{x})=\frac{1}{(2 \pi)^{d / 2}} \int d \mathbf{k} \sqrt{\frac{1}{2 \omega_{\mathbf{k}}}}\left[a^{+}(\mathbf{k}) e^{-i \mathbf{k} \mathbf{x}}+a^{-}(\mathbf{k}) e^{i \mathbf{k x}}\right]
\end{aligned}
$$

$\omega_{\mathbf{k}}=\sqrt{\mathbf{k}^{2}+m^{2}}$, причем в правой части уравнения (14) выбирается нормальное упорядочение, при котором операторы уничтожения $a^{-}$расставляются справа от операторов рождения $a^{+}$.

Рассмотрим построение приближенных решений уравнения (14) при $g \rightarrow 0$. Так как коммутатор операторов $\widehat{\pi}(\cdot) \sqrt{g}$ и $\widehat{\varphi}(\cdot) \sqrt{g}$, через которые выражается гамильтониан, пропорционален малому параметру $g$, то при $g \rightarrow 0$ применимы квазиклассические методы, в частности теория комплексного ростка [20-22].

Для дальнейшего нам будет удобнее рассмотреть несколько более общий случай, когда $H_{\Lambda, L}^{(0)}$ явно зависит от времени и имеет вид

$$
\frac{1}{g} H_{\Lambda, L}^{(0)}[\pi \sqrt{g}, \varphi \sqrt{g}]=\frac{1}{g} H_{0}[\pi \sqrt{g}, \varphi \sqrt{g}]+\xi(t) \frac{1}{g} H_{\Lambda, L}^{\mathrm{int}}[\pi \sqrt{g}, \varphi \sqrt{g}]
$$

где $\xi(t)$ - некоторая гладкая функция $t$. При $\xi(t)=1$ функционал (15) переходит в (13).

Рассмотрим унитарное преобразование

$$
U_{\Phi, \Pi, S}^{g}=e^{\frac{i}{g} S} \exp \left\{\frac{i}{\sqrt{g}} \int d \mathbf{x}[\Pi(\mathbf{x}) \widehat{\varphi}(\mathbf{x})-\Phi(\mathbf{x}) \widehat{\pi}(\mathbf{x})]\right\},
$$

где $S$ - вешественное число, $\Phi(\cdot), \Pi(\cdot)$ - вешественные функции. Из канонических коммутационных соотношений вытекает, что

$$
\begin{aligned}
\left(U_{\Phi, \Pi, S}^{g}\right)^{-1} \widehat{\varphi}(\mathbf{x}) U_{\Phi, \Pi, S}^{g} & =\widehat{\varphi}(\mathbf{x})+\Phi(\mathbf{x}) / \sqrt{g} \\
\left(U_{\Phi, \Pi, S}^{g}\right)^{-1} \widehat{\pi}(\mathbf{x}) U_{\Phi, \Pi, S}^{g} & =\widehat{\pi}(\mathbf{x})+\Pi(\mathbf{x}) / \sqrt{g} \\
\left(U_{\Phi, \Pi, S}^{g}\right)^{-1} \frac{d}{d t} U_{\Phi, \Pi, S}^{g} & =D_{t},
\end{aligned}
$$


где

$$
\begin{aligned}
D_{t}= & -\frac{1}{g}\left(\dot{S}^{t}+\frac{1}{2} \int d \mathbf{x}\left(\dot{\Pi}^{t}(\mathbf{x}) \Phi^{t}(\mathbf{x})-\dot{\Phi}^{t}(\mathbf{x}) \Pi^{t}(\mathbf{x})\right)\right)+ \\
& +\frac{1}{\sqrt{g}} \int d \mathbf{x}\left(\dot{\Phi}^{t}(\mathbf{x}) \widehat{\pi}(\mathbf{x})-\dot{\Pi}^{t}(\mathbf{x}) \widehat{\varphi}(\mathbf{x})\right)+d / d t .
\end{aligned}
$$

Рассмотрим элемент пространства $\mathcal{H}$ вида

$$
\Psi^{t}=U_{\Phi^{t}, \Pi^{t}, S^{t}}^{g} Y^{t}
$$

где вектор $Y^{t}$ имеет предел при $g \rightarrow 0$. Подстановка вектора (17) в уравнение (14) и использование правил коммутации (16) приводят к уравнению для $Y^{t}$ :

$$
i D_{t} Y^{t}=\sum_{n \geq 0} g^{n-1} H^{(n)}[\Pi(\cdot)+\sqrt{g} \widehat{\pi}(\cdot), \Phi(\cdot)+\sqrt{g} \widehat{\varphi}(\cdot)] Y^{t},
$$

индексы $\Lambda, L$ для краткости опушены.

Уравнение (18) содержит сингулярные при $g \rightarrow 0$ слагаемые порядка $O(1 / g)$ и $O(1 / \sqrt{g})$. Приравнивание этих слагаемых нулю приводит к соотношению

$$
\dot{S}^{t}=\frac{1}{2} \int d \mathbf{x}\left(-\dot{\Pi}^{t}(\mathbf{x}) \Phi^{t}(\mathbf{x})+\dot{\Phi}^{t}(\mathbf{x}) \Pi^{t}(\mathbf{x})\right)-H^{(0)}[\Pi(\cdot), \Phi(\cdot)]
$$

и системе уравнений Гамильтона

$$
\dot{\Phi}^{t}(\mathbf{x})=\frac{\delta H^{(0)}}{\delta \Pi(\mathbf{x})}, \quad \dot{\Pi}^{t}(\mathbf{x})=-\frac{\delta H^{(0)}}{\delta \Phi(\mathbf{x})} .
$$

При выполнении (19) и (20) уравнение (18) переходит при $g \rightarrow 0$ в уравнение

$$
i \frac{d}{d t} Y^{t}=H_{2}[\widehat{\pi}(\cdot), \widehat{\varphi}(\cdot)] Y^{t},
$$

где оператор $\widehat{H}_{2}=H_{2}[\widehat{\pi}(\cdot), \widehat{\varphi}(\cdot)]$ отвечает функционалу

$$
H_{2}[\pi(\cdot), \varphi(\cdot)]=\frac{1}{2} \pi \frac{\delta^{2} H^{(0)}}{\delta \Pi \delta \Pi} \pi+\pi \frac{\delta^{2} H^{(0)}}{\delta \Pi \delta \Phi} \varphi+\frac{1}{2} \varphi \frac{\delta^{2} H^{(0)}}{\delta \Phi \delta \Phi} \varphi+H^{(1)}
$$

Упорядочение операторов в $H_{2}[\widehat{\pi}(\cdot), \widehat{\varphi}(\cdot)]$ совпадает с упорядочением в $H[\widehat{\pi}(\cdot), \widehat{\varphi}(\cdot)]$. В формуле (22) аргументы $\Phi(\cdot), \Pi(\cdot)$ функционалов $H^{(0)}$ и $H^{(1)}$ опушены, а для ингералов типа

$$
\int \pi(\mathbf{x}) \frac{\delta^{2} H^{(0)}}{\delta \Pi(\mathbf{x}) \delta \Pi(\mathbf{y})} \pi(\mathbf{y}) d \mathbf{x} d \mathbf{y}
$$

введено краткое обозначение

$$
\pi \frac{\delta^{2} H^{(0)}}{\delta \Pi \delta \Pi} \pi
$$


Отметим, что уравнение Шредингера линеаризованной теории поля (21) также может быть получено с помощью выделения классической составляющей (2) непосредственно в регуляризованном действии (12) и канонического квантования теории поля с квадратичным действием (3).

Уравнение (21) имеет решение типа (6). Уравнение (7) для оператора $\widehat{M}^{t}$ и уравнение для $c^{t}$ могут быть представлены следуюшим образом:

$$
\begin{gathered}
\dot{\vec{M}}^{t}=\frac{1}{2}\left(1+\widetilde{M}^{t}\right) \frac{1}{\sqrt{\hat{\omega}}} \frac{\delta^{2} H^{(0)}}{\delta \Phi \delta \Phi} \frac{1}{\sqrt{\widehat{\omega}}}\left(1+\widetilde{M}^{t}\right)-\frac{1}{2}\left(1-\widetilde{M}^{t}\right) \widehat{\omega}\left(1-\widetilde{M}^{t}\right) \\
\left(\ln c^{t}\right)^{\cdot}=-\frac{i}{4}\left[\left(\frac{1}{\sqrt{\widehat{\omega}}} \frac{\delta^{2} H^{(0)}}{\delta \Phi \delta \Phi} \frac{1}{\sqrt{\widehat{\omega}}}-\widehat{\omega}\right) \widetilde{M}^{t}\right]-i H^{(1)}
\end{gathered}
$$

где $\widetilde{M}^{t}$ - оператор, ядро которого совпадает с преобразованием Фурье ядра $M^{t}(\mathbf{k}, \mathbf{p})$ оператора $\widehat{M}^{t}$ :

$$
\left(\widetilde{M}^{t} f\right)(\mathbf{x})=\int \frac{d \mathbf{k} d \mathbf{p} d \mathbf{y}}{(2 \pi)^{d}} e^{i(\mathbf{k x}+\mathbf{p y})} M^{t}(\mathbf{k}, \mathbf{p}) f(\mathbf{y}), \quad \widehat{\omega}=\sqrt{-\Delta+m^{2}}
$$

$\delta^{2} H^{(0)} /(\delta \Phi \delta \Phi)$ - оператор с ядром $\delta^{2} H^{(0)} /(\delta \Phi(\mathbf{x}) \delta \Phi(\mathbf{y}))$. После преобразования

$$
R=i \sqrt{\widehat{\omega}}(1-\widetilde{M})(1+\widetilde{M})^{-1} \sqrt{\widehat{\omega}}, \quad a^{t}=\frac{c^{t}}{(\operatorname{det}(1+\widetilde{M}))^{1 / 2}(\operatorname{det} \operatorname{Im} R)^{1 / 4}}
$$

уравнение (23) принимает к вид

$$
\dot{R}+R^{2}+\frac{\delta^{2} H^{(0)}}{\delta \Phi \delta \Phi}=0
$$

а уравнение (24) переходит в уравнение

$$
i\left(\ln a^{t}\right)^{\cdot}=-\frac{1}{2} \operatorname{Tr}\left(\operatorname{Im} R-\sqrt{-\Delta+m^{2}}-\frac{1}{2 \sqrt{-\Delta+m^{2}}} \frac{\delta^{2} H^{(0)}}{\delta \Phi \delta \Phi}\right)-H^{(1)} .
$$

Отметим, что преобразование (25) отвечает переходу от фоковского представления к шредингеровскому, в котором диагональны операторы поля.

Решение уравнения (21) с начальным условием

$$
P\left(a^{+}\right) \exp \left(\frac{1}{2} \int d \mathbf{k} a^{+}(\mathbf{k})\left(\widehat{M}^{0} a^{+}\right)(\mathbf{k})\right) \Phi^{(0)}
$$

где $P\left(a^{+}\right)$- полином по операторам рождения, строится с помошью ростковых операторов рождения [20]

$$
\Lambda\left[p^{t}, q^{t}\right]=\int d \mathbf{x}\left[p^{t}(\mathbf{x}) \widehat{\varphi}(\mathbf{x})-q^{t}(\mathbf{x}) \widehat{\pi}(\mathbf{x})\right]
$$


коммутируюших с оператором $i(d / d t)-\widehat{H}_{2}$, при этом

$$
\dot{p}^{t}(\mathbf{x})=-\frac{\delta H_{2}\left(p^{t}(\cdot), q^{t}(\cdot)\right)}{\delta q(\mathbf{x})}, \quad \dot{q}^{t}(\mathbf{x})=\frac{\delta H_{2}\left(p^{t}(\cdot), q^{t}(\cdot)\right)}{\delta p(\mathbf{x})} .
$$

Операторы (28) переводят одно решение уравнения (21) в другое.

\section{3. ПРОБЛЕМА СНЯТИЯ РЕГУЛЯРИЗАЦИИ В ГАМИЛЬТОНОВОМ ФОРМАЛИЗМЕ}

Рассмотрим теперь полученные квазиклассические уравнения при снятии регуляризации, т.е. в пределе $\Lambda \rightarrow \infty, L \rightarrow \infty$.

Обобшая данное во введении определение оператора (9), регулярного в рамках теории возмушений, дадим определение оператора, регулярного при $\Lambda, L \rightarrow \infty$ в главном порядке квазиклассического разложения. В соответствии с предыдушим разделом квазиклассические начальные условия сингулярным образом зависят от параметра $g$, имея вид (17). Естественно считать оператор $W_{\Lambda, L}^{t}$ регулярным при $\Lambda, L \rightarrow \infty$ в главном порядке квазиклассики, если параметры, входящие в квазиклассическую асимптотику вектора $W_{\Lambda, L}^{t} \Psi_{\Lambda, L}^{g}$, имеют предел при $\Lambda, L \rightarrow \infty$. Это значит, что для любой пары функций $\Phi^{0}(\mathbf{x}), \Pi^{0}(\mathbf{x})$ из пространства Шварца существует такая пара функций $\Phi_{\Lambda, L}^{0}(\mathbf{x}), \Pi_{\Lambda, L}^{0}(\mathbf{x})$ из этого же пространства и такое вешественное число $S^{t}$, что оператор

$$
V_{\Lambda, L, g}^{t}=\left(U_{\Phi_{\Lambda, L}^{t}, \Pi_{\Lambda, L}^{t}, S_{\Lambda, L}^{t}}^{g}\right)^{-1} W_{\Lambda, L, g}^{t} U_{\Phi^{0}, \Pi^{0}, 0}^{g}
$$

имеет сильньй предел при $g \rightarrow 0$, а предельные операторы

$$
\lim _{g \rightarrow 0}\left(U_{\Phi_{\Lambda, L}^{t}, \Pi_{\Lambda, L}^{t}, S_{\Lambda, L}^{t}}^{g}\right)^{-1} W_{\Lambda, L, g}^{t} U_{\Phi^{0}, \Pi^{0}, 0}^{g}
$$

сильно сходятся при $\Lambda, L \rightarrow \infty$. При выполнении этого свойства будем называть оператор $T_{\Lambda, L}$, входяший в формулу (9), одеваюшим преобразованием в главном порядке квазиклассического разложения.

Построим это преобразование с помошью процедуры "включения и выключения взаимодействия", которая использовалась Боголюбовым [23, 24] (см. также [25]) для устранения расходимостей Штюкельберга [26].

Рассмотрим бесконечно дифференцируемую функцию $\xi(\tau), \tau \leq 0$, обрашаюшуюся при $\tau<-T_{1}$ в нуль, а при $-T_{2}<\tau<0-$ в единицу (см. рисунок). Рассмотрим также оператор

$$
H_{\mathrm{int}}^{\Lambda, L}=\xi(\tau) \int d \mathbf{x} \chi(\mathbf{x} / L) \frac{1}{g} V_{\mathrm{int}}\left(\sqrt{g} \varphi^{\Lambda}(\mathbf{x})\right)+H_{\mathrm{ct}}(\sqrt{g} \widehat{\varphi}(\cdot), \sqrt{g} \widehat{\pi}(\cdot), \tau, \xi(\cdot)),
$$

причем при $\tau \in\left(-T_{2}, 0\right)$ функционал $H_{\mathrm{ct}}$ не зависит от $\tau$, а при $\tau<-T_{1}$ имеем $H_{\mathrm{ct}}=0$. Явньй вид $H_{\mathrm{ct}}$ приводится в следуюшем разделе. 


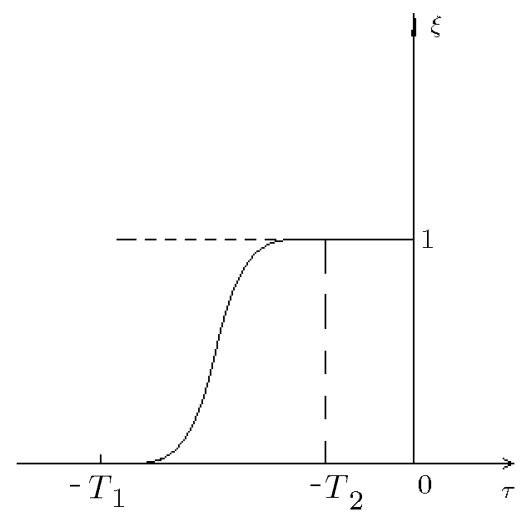

Функция включения взаимодействия

Обозначим через

$$
H_{0}=\int d \mathbf{k} \omega_{\mathbf{k}} a^{+}(\mathbf{k}) a^{-}(\mathbf{k})
$$

свободный гамильтониан. Выберем в качестве “одевающего преобразования” оператор вида

$$
T_{\Lambda, L}=T \exp \left[-i \int_{-\infty}^{0} d \tau e^{i H_{0} \tau} H_{\mathrm{int}}^{\Lambda, L}(\tau) e^{-i H_{0} \tau}\right]
$$

который переводит начальное условие задачи Коши

$$
\begin{aligned}
i \frac{d X^{\tau}}{d \tau} & =e^{i H_{0} \tau} H_{\mathrm{int}}^{\Lambda, L}(\tau) e^{-i H_{0} \tau} X^{\tau}, \\
X^{-\infty} & =X
\end{aligned}
$$

в решение этой задачи при $\tau=0$. Вследствие финитности функции $\xi$ правая часть уравнения (33) обрашается в нуль при $\tau<-T_{1}$. Поэтому начальное условие при $\tau=-\infty$ можно заменить начальным условием при $\tau=-T_{0}<-T_{1}$. Отметим, что данньй выбор преобразования $T_{\Lambda, L}$ согласуется с процедурой построения уравнения Шредингера, предложенной в [24].

Найдем в квазиклассическом приближении вектор (10) и оператор (9). Введем для этого вспомогательные обозначения. Пусть $S$ и $i \ln a-$ вешественные числа, $\Phi(\mathbf{x}), \Pi(\mathbf{x})$, $q(\mathbf{x}), p(\mathbf{x})$ - гладкие, быстроубываюшие функции, $R$ - оператор с симметричным ядром и положительно-определенной мнимой частью.

Обозначим через $\mathcal{U}_{\xi(\cdot)}^{t_{2}, t_{1}}$ отображение, переводяшее начальные условия при $t=t_{1}$ задач Коши для уравнений (19), (20), (26), (27), (29):

$$
p^{t_{1}}=p, \quad q^{t_{1}}=q, \quad \Phi^{t_{1}}=\Phi, \quad \Pi^{t_{1}}=\Pi, \quad R^{t_{1}}=R, \quad S^{t_{1}}=S, \quad a^{t_{1}}=a,
$$

в решения этих уравнений при $t=t_{2}$

$$
\left(p^{t_{2}}, q^{t_{2}}, \Phi^{t_{2}}, \Pi^{t_{2}}, R^{t_{2}}, S^{t_{2}}, a^{t_{2}}\right)=\mathcal{U}_{\xi(\cdot)}^{t_{2}, t_{1}}\left(p^{t_{1}}, q^{t_{1}}, \Phi^{t_{1}}, \Pi^{t_{1}}, R^{t_{1}}, S^{t_{1}}, a^{t_{1}}\right)
$$


где $\xi(\cdot)$ - функция включения взаимодействия, входяшая в формулу (15).

Для построения вектора $T_{\Lambda, L} X^{-\infty}$ при $g \rightarrow 0$ проведем замену $\Psi^{t}=e^{-i H_{0} \tau} X^{\tau}$ в уравнении (33). Задача Коши (33) приводится к виду

$$
\begin{aligned}
i \frac{d \Psi^{\tau}}{d \tau} & =\left[H_{0}+H_{\mathrm{int}}^{\Lambda, L}(\tau)\right] \Psi^{\tau}, \\
\Psi^{-T_{0}} & =e^{i H_{0} T_{0}} X^{-\infty}
\end{aligned}
$$

Выберем начальное условие $X^{-\infty}$ следуюшим образом:

$$
\begin{aligned}
X_{g}^{-\infty}= & U_{\Phi, \Pi, 0}^{g} c \Lambda\left[p_{1}(\cdot), q_{1}(\cdot)\right] \ldots \Lambda\left[p_{k}(\cdot), q_{k}(\cdot)\right] \times \\
& \times \exp \left(\frac{1}{2} \int d \mathbf{k} a^{+}(\mathbf{k})\left(M a^{+}\right)(\mathbf{k})\right) \Phi^{(0)}
\end{aligned}
$$

где $M$ - оператор Гильберта-Шмидта с нормой меньше единицы, $\Phi$, П, $c, M$ регулярны при $\Lambda, L \rightarrow \infty$, причем оператор $M$ и в пределе $\Lambda, L \rightarrow \infty$ остается оператором Гильберта-Шмидта.

В соответствии с предыдушим разделом асимптотическое решение задачи Коши (34) при $\tau=0$ есть

$$
\begin{aligned}
\Psi_{g}^{0}= & U_{\Phi^{\prime}, \Pi^{\prime}, S^{\prime}}^{g} c^{\prime} \Lambda\left[p_{1}^{\prime}(\cdot), q_{1}^{\prime}(\cdot)\right] \ldots \Lambda\left[p_{k}^{\prime}(\cdot), q_{k}^{\prime}(\cdot)\right] \times \\
& \times \exp \left(\frac{1}{2} \int d \mathbf{k} a^{+}(\mathbf{k})\left(M^{\prime} a^{+}\right)(\mathbf{k})\right) \Phi^{(0)},
\end{aligned}
$$

где зависящие от $\Lambda, L$ параметры, входящие в формулу (35), имеют вид

$$
\begin{aligned}
\left(p_{j}^{\prime}, q_{j}^{\prime}, \Phi^{\prime}, \Pi^{\prime}, R\left(M^{\prime}\right), S^{\prime}, a\left(c^{\prime}\right)\right) & =\mathcal{U}_{\xi(\cdot)}^{0,-T_{0}} \mathcal{U}_{0}^{-T_{0}, 0}\left(p_{j}, q_{j}, \Phi, \Pi, R(M), 0, a(c)\right), \\
j & =\overline{1, k},
\end{aligned}
$$

через $R(M)$ и $а(c)$ обозначены величины $(25)$.

Поскольку уравнения (19), (20), (29) регулярны при $\Lambda, L \rightarrow \infty$, параметры $\Phi^{\prime}, \Pi^{\prime}, S^{\prime}$, $p_{j}^{\prime}, q_{j}^{\prime}$ также имеют конечные пределы при снятии регуляризации.

Оператор $M^{\prime}$ является решением уравнения (7) и удовлетворяет начальному условию $M^{-T_{0}}$ при $t=-T_{0}$, предел которого при $\Lambda, L \rightarrow \infty$ представляет собой оператор Гильберта-Шмидта. Однако предел при $\Lambda, L \rightarrow \infty$ оператора $M^{\prime}$ может и не быть оператором Гильберта-Шмидта.

Таким образом, гауссовский вектор (17) регуляризованной теории

$$
U_{\Phi, \Pi, 0}^{g} \text { const } \exp \left(\frac{1}{2} \int d \mathbf{k} a^{+}(\mathbf{k})\left(M a^{+}\right)(\mathbf{k})\right) \Phi^{(0)}
$$

отвечает элементу перенормированного пространства Фока не тогда, когда $M$ является оператором Гильберта-Шмидта при снятии обрезаний, а тогда, когда решение уравнения (23), совпадаюшее с $\widetilde{M}$ при $t=0$, становится оператором Гильберта-Шмидта при 
$t<-T_{1}$. Константа в формуле (36), вообще говоря, нерегулярна при $\Lambda, L \rightarrow \infty$ и определется из следуюшего условия: решение уравнения $(24)$, совпадаюшее при $t=0$ с этой константой, имеет для $t<-T_{1}$ предел при $\Lambda, L \rightarrow \infty$.

Переформулируем это утверждение в терминах оператора $R$. Пусть упорядоченный символ оператора $\widetilde{M}$,

$$
\widetilde{M}=\widetilde{M}\left(\underset{\mathbf{x}}{2},-i \frac{\partial}{\partial \mathbf{x}}\right)
$$

убывает при $t \rightarrow-\infty$ как $\widetilde{M}(\mathbf{x}, \mathbf{k})=O\left(|\mathbf{k}|^{-d / 2-\delta}\right)$. При $\delta>0$ оператор $M$ является оператором Гильберта-Шмидта, при $\delta \leq 0$ - не является. Из формулы (25) вытекает, что символ оператора $R$ ведет себя на бесконечности как

$$
R(\mathbf{x}, \mathbf{k})=i \sqrt{\mathbf{k}^{2}+m^{2}}+O\left(|\mathbf{k}|^{-d / 2-\delta+1}\right) .
$$

При $\Lambda, L \rightarrow \infty$ уравнение (26) переходит в уравнение

$$
\dot{R}+R^{2}+\left[-\Delta+m^{2}+\xi(t) V_{\text {int }}^{\prime \prime}\left(\Phi_{c}(t, \mathbf{x})\right)\right]=0 .
$$

Полученный результат можно сформулировать следуюшим образом.

ПрЕДЛОЖЕнИЕ 1. Вектор (36) регуляризованной теории отвечает вектору перенормированного пространства состояний, если решение уравнения (38), равное $R$ при $t=0$, удовлетворяет при $t<-T_{1}$ свойству (37) для $\delta>0$. Если же свойство (37) при $t<-T_{1}$ выполнено для $\delta \leqslant 0$, то выражение (36) не отвечает никакому вектору перенормированного пространства состояний.

В следуюшем разделе мы сформулируем условия этого предложения явно, исследовав уравнение (38).

Рассмотрим теперь вопрос о сильной сходимости оператора (31) на плотной области определения. Любой вектор пространства Фока может быть аппроксимирован с любой точностью конечной линейной комбинацией векторов

$$
Y=c \Lambda\left[p_{1}(\cdot), q_{1}(\cdot)\right] \ldots \Lambda\left[p_{k}(\cdot), q_{k}(\cdot)\right] \Phi^{(0)} .
$$

Исследуем сходимость вектора

$$
\lim _{g \rightarrow 0} V_{\Lambda, L, g}^{t} Y
$$

при $\Lambda, L \rightarrow \infty$. Квазиклассическое асимптотическое представление для вектора

$$
W_{\Lambda, L, g}^{t} U_{\Phi^{0}, \Pi^{0}, 0}^{g} Y=\left(T_{\Lambda, L}\right)^{-1} U_{\Lambda, L}^{t} T_{\Lambda, L} U_{\Phi^{0}, \Pi^{0}, 0}^{g} Y
$$

строится в соответствии с предыдушим разделом и имеет вид $U_{\Phi^{\prime}, \Pi^{\prime}, S^{\prime}}^{g} Y^{\prime}$, где

$$
\begin{aligned}
Y^{\prime}= & c^{\prime} \Lambda\left[p_{1}^{\prime}(\cdot), q_{1}^{\prime}(\cdot)\right] \ldots \Lambda\left[p_{k}^{\prime}(\cdot), q_{k}^{\prime}(\cdot)\right] \times \\
& \times \exp \left(\frac{1}{2} \int d \mathbf{k} a^{+}(\mathbf{k})\left(M^{\prime} a^{+}\right)(\mathbf{k})\right) \Phi^{(0)} .
\end{aligned}
$$


Входящие в вектор (40) параметры имеют вид

$$
\begin{aligned}
& \left(p_{j}^{\prime}, q_{j}^{\prime}, \Phi^{\prime}, \Pi^{\prime}, R\left(M^{\prime}\right), S^{\prime}, a\left(c^{\prime}\right)\right)= \\
& \quad=\mathcal{U}_{0}^{0,-T_{0}} \mathcal{U}_{\xi(\cdot)}^{-T_{0}, 0} \mathcal{U}_{\xi(\cdot)}^{t,-T_{0}} \mathcal{U}_{0}^{-T_{0}, 0}\left(p_{j}, q_{j}, \Phi^{0}, \Pi^{0}, R(0), 0, a(c)\right), \quad j=\overline{1, k},
\end{aligned}
$$

а функция $\xi(t)$ при $t>0$ продолжается так, что $\xi(t)=1$. Если $\Phi_{\Lambda, L}^{t}=\Phi^{\prime}, \Pi_{\Lambda, L}^{t}=\Pi^{\prime}$, $S_{\Lambda, L}^{t}=S^{\prime}$, то вектор (39) совпадает с (40).

Поскольку $p_{j}^{\prime}, q_{j}^{\prime}$ регулярны при $\Lambda, L \rightarrow \infty$, для доказательства регулярности вектора (40) при $\Lambda, L \rightarrow \infty$ достаточно показать, что:

a) $M^{\prime}$ - оператор Гильберта-Шмидта,

б) $c^{\prime}$ имеет предел при $\Lambda, L \rightarrow \infty$.

Требование "б" равносильно при условии "а" требованию конечности величины $\ln a^{\prime}=\ln a\left(c^{\prime}\right)$. Условие "а" означает, что для символа оператора $R^{\prime}=R\left(M^{\prime}\right)$ выполнено свойство (37).

Исследуем уравнение (38) и покажем, что при определенном выборе контрчлена $H^{(1)}$ условия "а" и "б" выполняются.

\section{4. УСЛОВИЕ НА ГАУССОВСКИЙ ВЕКТОР СОСТОЯНИЯ}

Рассмотрим уравнение (38). Обозначим через $R(\mathbf{x}, \mathbf{k})$ упорядоченный символ оператора $R$ :

$$
R=R\left(\stackrel{2}{\mathbf{x}},-i \frac{\partial}{\partial \mathbf{x}}\right)
$$

Индексы 1 и 2 означают, что операторы дифференцирования расставлены справа от операторов умножения на $\mathbf{x}$ :

$$
R \int d \mathbf{k} f(\mathbf{k}) e^{i \mathbf{k} \mathbf{x}} \equiv \int d \mathbf{k} R(\mathbf{x}, \mathbf{k}) f(\mathbf{k}) e^{i \mathbf{k} \mathbf{x}} .
$$

В этом разделе мы исследуем поведение символа $R(\mathbf{x}, \mathbf{k})$ при $\mathbf{k} \rightarrow \infty$.

Рассмотрим асимптотическое разложение этого символа по $1 / \omega_{\mathbf{k}}, \omega_{\mathbf{k}}=\sqrt{\mathbf{k}^{2}+m^{2}}$ при $\mathbf{k} \rightarrow \infty$ :

$$
R^{t}(\mathbf{x}, \mathbf{k})=\mathcal{R}^{t}(\mathbf{x}, \mathbf{k})=i \omega_{\mathbf{k}}+\sum_{m \geq 1} \frac{R_{m}^{t}\left(\mathbf{x}, \mathbf{k} / \omega_{\mathbf{k}}\right)}{\omega_{\mathbf{k}}^{m}},
$$

где $R_{m}^{t}(\mathbf{x}, \mathbf{n})$ - гладкие функции, быстроубывающие по х на бесконечности вместе с производными всех порядков. Мы подставим разложение (42) в уравнение (38) и найдем, при каких условиях (38) будет выполнено с точностью $O\left(1 / \omega_{\mathbf{k}}^{j}\right)$, где $j$ - произвольное заданное число. Воспользуемся формулой для упорядоченного символа произведения операторов $[27,28]$.

Пусть

$$
\widehat{A}=A\left(\stackrel{2}{\mathbf{x}},-i \frac{\partial}{\partial \mathbf{x}}\right), \quad \widehat{B}=B\left(\stackrel{2}{\mathbf{x}},-i \frac{\partial}{\partial \mathbf{x}}\right)
$$


- некоторые операторы. Тогда их произведение $\widehat{A} \widehat{B}$ имеет упорядоченный символ $A * B$,

$$
\widehat{A} \widehat{B}=(A * B)\left(\stackrel{2}{\mathbf{x}},-i \frac{\partial}{\partial \mathbf{x}}\right),
$$

который представляется в одной из двух эквивалентных форм:

$$
\begin{aligned}
& (A * B)(\mathbf{x}, \mathbf{k})=A\left(\stackrel{2}{\mathbf{x}}, \mathbf{k}-i \frac{\partial}{\partial \mathbf{x}}\right) B(\mathbf{x}, \mathbf{k}), \\
& (A * B)(\mathbf{x}, \mathbf{k})=\int \frac{d \mathbf{z} d \mathbf{p}}{(2 \pi)^{d}} A(\mathbf{x}, \mathbf{k}-\mathbf{p}) B(\mathbf{x}+\mathbf{z}, \mathbf{k}) e^{i \mathbf{p z}} .
\end{aligned}
$$

Для вывода формулы (44) достаточно воспользоваться соотношением

$$
\widehat{B} \int d \mathbf{p} f(\mathbf{p}) e^{i \mathbf{p} \mathbf{x}}=\int d \mathbf{k} e^{i \mathbf{k x}} \int \frac{d \mathbf{z} d \mathbf{p}}{(2 \pi)^{d}} e^{i(\mathbf{p}-\mathbf{k}) \mathbf{y}} B(\mathbf{y}, \mathbf{p}) f(\mathbf{p}),
$$

вытекаюшим из формулы обрашения для преобразования Фурье и формулы (41), и применить к нему оператор $\widehat{A}$. Формула (43) следует из (44).

Раскладывая формально правую часть формулы (43) в ряд по оператору дифференцирования, получаем

$$
(A * B)(\mathbf{x}, \mathbf{k})=\sum_{l \geq 0} \frac{(-i)^{l}}{l !} \frac{\partial^{l} A(\mathbf{x}, \mathbf{k})}{\partial k_{i_{1}} \ldots \partial k_{i_{l}}} \frac{\partial^{l} B(\mathbf{x}, \mathbf{k})}{\partial x_{i_{1}} \ldots \partial x_{i_{l}}},
$$

где по повторяюшимся индексам подразумевается суммирование. В случае, если функции $A$ и $B$ имеют вид

$$
A=\frac{A_{1}^{t}\left(\mathbf{x}, \mathbf{k} / \omega_{\mathbf{k}}\right)}{\omega_{\mathbf{k}}^{m_{1}}}, \quad B=\frac{B_{1}^{t}\left(\mathbf{x}, \mathbf{k} / \omega_{\mathbf{k}}\right)}{\omega_{\mathbf{k}}^{m_{2}}}
$$

ряд (45) является асимптотическим разложением по $1 / \omega_{\mathbf{k}}$, поскольку $l$-е слагаемое формулы (45) имеет порядок $O\left(\omega_{\mathbf{k}}^{-m_{1}-m_{2}-l}\right)$ при $\mathbf{k} \rightarrow \infty$. Обоснование асимптотической формулы (45) может быть получено применением метода стационарной фазы [29] к интегралу (44).

Используя формулы (45) и (42), подставляя символ $\mathcal{R} * \mathcal{R}$ оператора $\mathcal{R}^{2}$ в уравнение (38) и приравнивая нулю слагаемые порядка $O\left(1 / \omega_{\mathbf{k}}^{s}\right), s \geq 0$, получаем соотношение

$$
2 i R_{1}^{t}(\mathbf{x}, \mathbf{n})+\xi(t) V_{\text {int }}^{\prime \prime}\left(\Phi^{t}(\mathbf{x})\right)=0
$$

при $s=0$ и соотношение

$$
\begin{gathered}
\dot{R}_{s}^{t}(\mathbf{x}, \mathbf{n})+2 i R_{s+1}^{t}(\mathbf{x}, \mathbf{n})+\sum_{\substack{l+m=s+1 \\
l, m \geq 1}} \omega_{\mathbf{k}}^{l-1} \frac{(-i)^{l-1}}{l !} \frac{\partial^{l} \omega_{\mathbf{k}}}{\partial k_{i_{1}} \ldots \partial k_{i_{l}}} \frac{\partial^{l} R_{m}^{t}(\mathbf{x}, \mathbf{n})}{\partial x_{i_{1}} \ldots \partial x_{i_{l}}}+ \\
\quad+\sum_{\substack{m+n+l=s \\
m, n \geq 1, l \geq 0}} \frac{(-i)^{l}}{l !} \omega_{\mathbf{k}}^{m+l} \frac{\partial^{l}}{\partial k_{i_{1}} \ldots \partial k_{i_{l}}} \frac{R_{m}^{t}\left(\mathbf{x}, \mathbf{k} / \omega_{\mathbf{k}}\right)}{\omega_{\mathbf{k}}^{m}} \frac{\partial^{l} R_{n}^{t}(\mathbf{x}, \mathbf{n})}{\partial x_{i_{1}} \ldots \partial x_{i_{l}}}=0
\end{gathered}
$$


при $s \geq 1$; в этих выражениях $\mathbf{n}=\mathbf{k} / \omega_{\mathbf{k}}$. При выводе формулы (46) использовано предположение, что $\dot{R}_{s}^{t}$ имеет тот же порядок, что и $R_{s}^{t}$.

Рекуррентное соотношение (46) позволяет выразить функцию $R_{s+1}^{t}$ через функции $R_{1}^{t}, \ldots, R_{s}^{t}$, найденные в предыдущих порядках теории возмушений по $1 / \omega_{\mathbf{k}}$. При этом $R_{s+1}^{t}$ однозначно определяется значениями полей $\Phi^{t}(\cdot)$, импульсов $\Pi^{t}(\cdot)$ и значениями функции $\xi$ и ее производных в момент времени $t$.

При $\xi(t)=0$, т.е. $t<-T_{1}$, полученные коэффициенты асимптотического разложения обрашаются в нуль, так что $R^{t}=i \omega_{\mathbf{k}}$ при этих значениях $t$. В случае $t>-T_{2}$ асимптотическая формула не зависит от $\xi$.

Приведем несколько первых функций $R_{s}^{t}$, определяемых из рекуррентных соотношений (46):

$$
\begin{aligned}
& R_{1}^{t}(\mathbf{x}, \mathbf{n})=\frac{i}{2} \xi(t) V_{\text {int }}^{\prime \prime}\left(\Phi^{t}(\mathbf{x})\right) \\
& R_{2}^{t}(\mathbf{x}, \mathbf{n})=-\frac{1}{4}\left(\frac{\partial}{\partial t}+\mathbf{n} \frac{\partial}{\partial \mathbf{x}}\right) \xi(t) V_{\mathrm{int}}^{\prime \prime}\left(\Phi^{t}(\mathbf{x})\right)
\end{aligned}
$$

Отметим, что первая производная $\Phi^{t}$ по времени выражается через импульс $\Pi^{t}$, а производные по времени от $\Phi^{t}$ высших порядков, входяшие в последуюшие функции $R_{s}$, находятся из уравнений движения через $\Phi^{t}$ и $\Pi^{t}$.

Мы построили асимптотическоепри $|\mathbf{k}| \rightarrow \infty$ разложение только для одного решения уравнения (38), которое при $t<-T_{1}$ отвечает вакууму, т.е. равно $i \omega_{k}$. Рассмотрим построение асимптотического решения уравнения (38), удовлетворяюшего начальному условию (37). Подставляя в уравнение (38) оператор с ядром $R^{t}=\mathcal{R}^{t}+r^{t}$, где $\mathcal{R}^{t}$ - уже построенное решение (42) уравнения (38), в главном порядке по $1 / \omega_{\mathbf{k}}$ получаем уравнение $\dot{r}^{t}+2 i \omega_{\mathbf{k}} r^{t}=0$. Его решение есть $r^{T}=r^{-T_{0}} \exp \left(-2 i \omega_{\mathbf{k}}\left(t+T_{0}\right)\right)$. Поправки к этому решению строятся в работе [30].

Таким образом, предложение 1 можно переформулировать следуюшим образом.

ПРЕДЛОЖЕНИЕ 2. Формула (36) отвечает вектору перенормированного пространства состояний тогда и только тогда, когда

$$
R^{0}=\mathcal{R}^{0}+O\left(|\mathbf{k}|^{-d / 2+1-\delta}\right)
$$

причем асимптотическое разложсение (42) для $\mathcal{R}^{0}$ не зависит от выбора функции включения взаимодействия $\xi(t)$ и определяется только функииями $\Pi^{0}(\mathbf{x})$ u $\Phi^{0}(\mathbf{x})$.

Остановимся на проверке условий, наложенных на $R^{\prime}$ и $a^{\prime}$ в конце предыдушего раздела.

Условие на $R^{\prime}$ сразу же вытекает из инвариантности при $t=0$ асимптотического разложения (42) относительно замены функции $\xi(t)$ на $\xi\left(t-t_{0}\right)$, где $t_{0}=$ const.

Величина $a^{\prime}$ является конечной в случае, если выбрать соответствуюшим образом контрчлен $H^{(1)}$, который компенсирует расходимости, возникаюшие при вычислении 
следа оператора $R$. Для нахождения явного вида этого контрчлена можно рассмотреть асимптотические решения регуляризованного уравнения (26)

$$
\mathcal{R}_{\Lambda, L}^{t}(\mathbf{x}, \mathbf{k})=i \omega_{\mathbf{k}}+\sum_{m \geq 1, j \geq 0} \frac{R_{m j}^{t}\left(\mathbf{x}, \mathbf{k} / \omega_{\mathbf{k}}, \mathbf{k} / \Lambda\right)}{\omega_{\mathbf{k}}^{m} \Lambda^{j}},
$$

выделить расходящуюся часть следа

$$
-\frac{1}{2} \operatorname{Tr}\left(\operatorname{Im} \mathcal{R}_{\Lambda, L}^{t}-i \omega_{k}\right) \sim-\frac{1}{2} \sum_{m=\overline{1, d}, j=\overline{0, d}} \int \frac{d \mathbf{k} d \mathbf{x} \operatorname{Im} R_{m j}^{t}\left(\mathbf{x}, \mathbf{k} / \omega_{\mathbf{k}}, \mathbf{k} / \Lambda\right)}{\omega_{\mathbf{k}}^{m} \Lambda^{j}},
$$

сложить ее с расходящейся частью следа

$$
\operatorname{Tr}\left(\frac{1}{4 \widehat{\omega}} \frac{\delta^{2} H^{(0)}}{\delta \Phi \delta \Phi}\right)
$$

и приравнять полученную сумму контрчлену $H^{(1)}$. Поскольку $R_{m j}^{t}$ однозначно определяется полями $\Phi^{t}(\cdot), \Pi^{t}(\cdot)$ и значением функции $\xi$ и ее производных в точке $t$, то контрчлен $H^{(1)}$ является при $t>-T_{2}$ функционалом от полей и импульсов. Тем самым требования, наложенные на $R^{\prime}$ и $a^{\prime}$, проверены.

\section{5. ЗАКЛЮЧЕНИЕ}

Таким образом, мы получили критерий того, что выражение (36) определяет элемент перенормированного пространства состояний (предложения 1 и 2).

Отметим, что в литературе встречались различные предположения о выборе начальных условий для уравнения (7). Так, в работах по квазиклассической теории поля, в которых не рассматривается проблема перенормировки (см., например, [7]), обычно неявно предполагается, что начальное условие для уравнения (26) при $t=0$ имеет вид (37). В статье [31] рассматривались начальные состояния, являюшиеся собственными для оператора Гамильтона в начальный момент времени. Этим состояниям отвечают гауссовские функционалы со следующей сингулярной частью символа оператора $R^{t}$ :

$$
(R * R)(\mathbf{x}, \mathbf{k}) \sim-\left(\mathbf{k}^{2}+m^{2}+V^{\prime \prime}\left(\Phi_{c}^{t}(\mathbf{x})\right)\right) .
$$

Формулы (37) и (48) получаются из нашего условия (47) только в том случае, если асимптотическое разложение при $|\mathbf{k}| \rightarrow \infty$ строится с точностью $O(1 /|\mathbf{k}|)$ и $O\left(1 /\left|\mathbf{k}^{2}\right|\right)$, соответственно, а это справедливо только для достаточно малых размерностей пространства. Вместе с тем для статических решений уравнений поля условие (48) справедливо всегда.

Хотя для простоты был рассмотрен только случай скалярной теории, аналогичные рассуж дения практически без изменений переносятся и на более сложные теории. Так, в случае калибровочной теории [32] можно взять калибровку типа кулоновской и записать в данной калибровке уравнение эволюции. После этого можно применить процедуру "включения и выключения взаимодействия" аналогично случаю скалярного поля. 
Вариант, когда теория, помимо бозонных, содержит и фермионные поля (в частности, квантовая электродинамика), также рассматривается аналогично. Так, подстановка (17), где оператор $U$ содержит только операторы бозонных полей, приближенно удовлетворяет уравнению Шредингера и при наличии фермионов. Вектор $Y^{t}$ при этом является решением фермионного уравнения Шредингера с квадратичным гамильтонианом, которое, так же как и бозонное, решается точно [11].

Полученное условие на оператор $R$ не зависит от контрчленов и определяется только классическим действием. Поэтому оно применимо как для перенормируемых, так и для неперенормируемых теорий.

Предэкспоненциальный множитель зависит от "однопетлевого" контрчлена $H^{(1)}$. Данный вывод согласуется с рассуждениями, изложенными в работе [32]: древесные диаграммы при вычислении континуального интеграла по методу стационарной фазы определяют показатель экспоненты, а однопетлевые - предэкспоненту.

Часто считается, что проблему расходимостей и перенормировок в теории поля следует учитывать только при рассмотрении диаграмм Фейнмана с петлями, а при исследовании древесных диаграмм проблем с расходимостями нет. Мы видим, что и в классической теории поля следует учитывать проблему расходимостей, поскольку именно из анализа этой проблемы мы и получили не зависящее от контрчленов условие на начальные данные для уравнения (26).

Мы рассмотрели только главный порядок квазиклассики. Исследование проблемы начальных условий для высших порядков разложения производится аналогично. Следует, однако, учесть, что, помимо ультрафииолетовых расходимостей, в высших порядках разложения возникают и объемные расходимости, связанные с рождением частиц из вакуума в соответствии с теоремой Хаага $[33,34]$. Поэтому взамен преобразования (32) следует взять преобразование, являюшееся композицией преобразования (32) и преобразования, устраняющего вакуумные расходимости [18].

Проблема регулярности оператора (30) в высших порядках разложения может быть сведена к проблеме регулярности $S$-матрицы Боголюбова $S(g)$ [24], отвечаюшей финитной функции включения и выключения взаимодействия. В случае, если классическое решение тождественно равно нулю, данное утверждение вытекает из теоремы Боголюбова-Парасюка $[24,35,36]$. Мы рассмотрим эти вопросы подробно в последующих публикациях.

Работа выполнена при финансовой поддержке Российского фонда фундаментальных исследований, проект 96-01-01544.

\section{Список литературы}

[1] R. Dashen, B. Hasslasher, A. Neveu. Phys. Rev. D. 1974. V. 10. P. 4114.

[2] L.D. Faddeev, V.E. Korepin. Phys. Rep. 1978. V. 42. P. 1.

[3] Р. Раджараман. Солитоны и инстантоны в квантовой теории поля. М.: Мир, 1985.

[4] А. А. Гриб, С. Г. Мамаев, В. М. Мостепаненко. Вакуумные квантовые эффекты в сильных полях. М.: Энергоатомиздат, 1988.

[5] Н. Биррелл, П. Девис. Квантовые поля в искривленном пространстве-времени. М.: Мир, 1984. 
[6] Д. М. Гитман, Е. С. Фрадкин, ШІ. М. Швариман. Квантовая электродинамика с нестабильным вакуумом. М.: Наука, 1991.

[7] В. Г. Багров, Д. М. Гитман, В.А. Кучин. Актуальные проблемы теоретической физики. Ред. А.А. Соколов. М.: МГУ, 1976.

[8] F. Cooper, E. Mottola. Phys.Rev.D. 1987. V. 36. P. 3114.

[9] S.-Y. Pi, M. Samiullah. Phys. Rev. D. 1987. V. 36. P. 3128.

[10] O. Eboli, S.-Y. Pi, M. Samiullah. Ann. Phys. 1989. V. 193. P. 102.

[11] Ф. А. Березин. Метод вторичного квантования. М.: Наука, 1986.

[12] А.А. Гриб, С. Г. Мамаев. ЯФ. 1969. Т. 10. С. 1276.

[13] О.И. Завьялов, В.Н. Сушко. В сб: Статистическая физика и квантовая теория поля. Ред. Н. Н. Боголюбов. М.: Наука, 1973.

[14] K. Xenn. Теория перенормировок. М.: Наука, 1974.

[15] Джс. Глимм, А. Джаффе. В сб: Конструктивная теория поля. Ред. В. Н. Сушко. М.: Мир, 1977.

[16] И. Я. Арефьева. ТМФ. 1973. Т. 14. № 1. С. 3.

[17] И. Я. Арефьева. ТМФ. 1973. Т. 15. № 2. С. 207.

[18] Л. Д. Фаддеев. ДАН СССР. 1963. Т. 152. С. 573.

[19] А. С. Швари. Математические основы квантовой теории поля. М.: Атомиздат, 1975.

[20] В. П. Маслов. Комплексный метод ВКБ в нелинейных уравнениях. М.: Наука, 1977.

[21] В. П. Маслов, О. Ю. Шведов. ТМФ. 1995. Т. 104. № 2. С. 310.

[22] В. П. Маслов, О. Ю. Шведов. ТМФ. 1995. Т. 104. № 3. С. 479.

[23] Н. Н. Боголюбов. ДАН СССР. 1951. Т. 81. С. 757.

[24] Н. Н. Боголюбов, Д. В. Ширков. Введение в теорию квантованных полей. М.: Наука, 1973.

[25] А. Д. Суханов. ЖЭЭТ. 1962. Т. 43. С. 932.

[26] E. C. G. Stueckelberg. Phys.Rev. 1951. V. 81. P. 130.

[27] М. В. Карасев, В. П. Маслов. Нелинейная скобка Пуассона. Геометрия и квантование. М.: Наука, 1991.

[28] В. П. Маслов. Операторные методы. М.: Наука, 1973.

[29] В. П. Маслов. Теория возмущений и асимптотические методы. М.: МГУ, 1965.

[30] В. П. Маслов, О. Ю. Шведов. Докл. РАН. 1998. Т. 358. № 3.

[31] М. И. Широков. ЯФ. 1968. Т. 7. С. 672.

[32] А.А. Славнов, Л.Д. Фаддеев. Введение в квантовую теорию калибровочных полей. М.: Наука, 1988.

[33] A. Вайтман. Проблемы в релятивистской динамике квантованных полей. М.: Наука, 1968.

[34] Н. Н. Боголюбов, А. А. Логунов, А. И. Оксак, И. Т. Тодоров. Общие принципы квантовой теории поля. М.: Наука, 1987.

[35] О. И. Завьялов. Перенормированные диаграммы Фейнмана. М.: Наука, 1979.

[36] A. С. Швари. Элементы квантовой теории поля. Бозонные взаимодействия. М.: Атомиздат, 1975 . 